\title{
Dark matter as a condensate: Deduction of microscopic properties
}

\author{
S. Gutiérrez • B. Carvente • A. Camacho
}

\begin{abstract}
In the present work we model dark matter as a Bose-Einstein condensate and the main goal is the deduction of the microscopic properties, namely, mass, number of particles, and scattering length, related to the particles comprised in the corresponding condensate. This task is done with the introduction, in the corresponding model, of the effects of the thermal cloud of the system. Three physical conditions are imposed, i.e., mechanical equilibrium of the condensate, explanation of the rotation curves of stars belonging to some galaxies, and, finally, the deflection of light due to the presence of dark matter. These three aforementioned expressions allow us to cast the features of the particles in terms of detectable astrophysical variables. Finally, the model is contrasted against the observational data of fourteen galaxies and in this manner we obtain values for the involved microscopic parameters of the condensate. The statistical errors are five and twenty four percent for the scattering length and mass of the dark matter particle, respectively.
\end{abstract}

Keywords dark matter, gravitation

\section{Introduction}

The current astrophysical observations allow us to conclude that more than 27 percent of the content of the universe is comprised by dark matter, dark energy near 68 percent, and baryons a 5 percent (Binney and Tremaine 1987; Planck Collaboration

\footnotetext{
S. Gutiérrez

B. Carvente

A. Camacho

Departamento de Física, Universidad Autónoma MetropolitanaIztapalapa

Apartado Postal 55-534, C.P. 09340, México, D.F., México.
}

2016). The conundrum appears when we note that we do not see or understand the first two components. Here the phrase dark matter can be understood as invisible, cold, and almost pressureless matter distributed around the galaxies. In the context of dark matter we may state that, in the gravitational realm, it plays a dominant role, in other words, though it is a fundamental element in the description of the Universe the knowledge that we have of it is very scarce.

As mentioned before the corresponding evidence of its presence emerges from astronomy (Reid et al 2010) and the rotation curves of galaxies provide one of the best examples of its existence. Astronomical observations do attest that the rotational velocities increase near the geometric center of the corresponding galaxy and that these velocities remain almost constant. This last result entails a mass profile for this kind of galaxies such that it does not coincide with the content of baryonic mass.

There are very few facts that we know about it and they can be mentioned very briefly. Indeed, if it were comprised by baryons the cosmic microwave background would have a completely different structure from the one observed, i.e., it cannot consist of baryons. We must also discard light particles (not created by a phase transition in the early Universe) (Viel et al 2005; Abazajian 2006) and the lower bound for the mass of the corresponding particle is $m>2 \mathrm{Kev}$. In addition, it has neither an electric charge nor a magnetic dipole moment.

The lack of a deeper comprehension of the constituents of dark matter has spurred a large number of models for its explanation. Among the candidates we may find WIMPS (weakly-interacting massive particles) (Steigman and Turner 1985), axions (Kolb and Turner 1990), etc. For a review on this topic see (Chavanis 2011) and references therein. 
It is noteworthy to comment that among the aforementioned possibilities we may found the option related to a Bose-Einstein condensate (BEC) as a backbone description for dark matter (Baldeschi et al 1983). Here the physical meaning of a BEC is related to the fact that at very low temperatures most of the particles of a bosonic gas occupy the ground state of the corresponding single-particle Hamiltonian operator (Pethick and Smith 2004). In the case in which only two-body interactions are taken into account the BEC can be described resorting to the so-called GrossPitaevskii equation, a non-linear Schrödinger differential equation in which the non-linearity has a cubic structure consequence of the interaction among the particles of the gas (Ueda 2010).

The work in this direction includes the emergence of the seeds of galaxies as a consequence of the presence of the BEC (Nishivama et al 2004), a relativistic version of the Gross-Pitaevskii equation (Grifols 2006), BEC formed by neutrinos due to the assumption that they violate the Pauli Exclusion Principle (Dolgov and Smirnov 2005), etc. For a deeper look at the literature in this context see (Böhmer and Harko 2007).

It has to be emphatically underlined that in the deduction of the Gross-Pitaevskii equation several assumptions are introduced, namely, we neglect interactions between degrees of freedom related to length scales smaller than the average interparticle spacing, and all atoms are in the ground state of the corresponding Hamiltonian, this is the so-called Hartree approximation (Pethick and Smith 2004), only pairwise interactions are relevant. These conditions imply that there are no particles in excited states, only the ground state is populated. A few words concerning our model have to be said at this point. Usually in the analogy between dark matter and BEC (Chavanis 2011; Böhmer and Harko 2007) the assumptions made involve that all particles are in the ground state and that there is a scattering length which is not necessarily null. The theory of ultra-cold gasses shows us that if there is a non-vanishing scattering length, then, inexorably, the number of particles populating excited states cannot be zero (Pitaevskii and Stringari 2003), the so-called depletion term appears. In the present work we take into account the thermal cloud and analyze its consequences.

The mathematical model is defined by GrossPitaevskii-Poisson system (see below) (Chavanis 2011; Böhmer and Harko 2007) the one, in the realm of analytical results, is usually accompanied by the Gaussian Ansatz, i.e., an approximate expression for the density profile is given in the form of a Gaussian function.
In the present work we will provide an argument which explains the physics behind this assumption as a consequence of the fact that in the roughest approximation the self-gravitational interaction of the halo can be understood as the case of a BEC immersed in an isotropic harmonic oscillator trap. It will be shown that if we follow this line of argumentation we may recover, immediately, the radius of a BEC (for the case in which no scattering length is present) without imposing any additional requirement. This interpretation will endow the system with an effective trapping potential and, in consequence, we will deduce the energy of the gas assuming that only the ground and the first excited states are populated.

The extant work in this context usually considers the consequences of a BEC upon the rotation curves of galaxies, the appearance of gravitational instabilities, etc. In other words, the microscopic properties of the BEC are assumed and starting from this primordial assumption the corresponding consequences upon diverse physical scenarios are obtained. It has to be underlined the fact that (as far as the authors know) there is a void in the literature in this topic. Indeed, there are no works in which the microscopic properties of a BEC, depicting dark matter, are deduced from the current observational data. This is a task that has to be done since amidst the scarce information concerning dark matter at our disposition we may find a lower bound for the mass of the involved dark matter particle. This last comment defines the main goal of the present work, namely, the deduction of the microscopic properties (mass, scattering length and number of particles) in a BEC in the context of dark matter. Here we do not assume anything about the mass or scattering length of the dark matter particles, but deduce them from the current observational data.

Since three physical parameters are to be obtained we need, at least, three independent expressions relating the microscopic properties to variables obtained by observational data. In order to have these required expressions we impose three physical conditions upon our BEC: (i) mechanical equilibrium of the condensate; (ii) concordance of the model with the rotation curves of stars belonging to some galaxies, and, finally; the deflection of light due to the presence of dark matter. Let us now explain, briefly, how we will proceed. Firstly, the energy of the gas will be calculated and with it the condition of mechanical equilibrium deduced, a fact leading us to a relationship defining the radius of the BEC as a function of the mass and scattering length of the dark matter particles and, also as a function of the number of particles in the condensate. Secondly, we relate the tangential velocity of galaxies with the matter 
content of our halo. Finally, we consider a light beam moving toward the halo and the ensuing deflection due to the mass of the condensate is obtained. Clearly, the model contains three unknown features related to the BEC, i.e., mass and scattering length of the dark matter particles plus the number of particles in the gas and our work includes three features which can be detected by astrophysical and astronomical means and depending upon our BEC characteristics.

We apply our model to the case of some galaxies and find that the mass and scattering length of the dark matter particle are, $m \sim 10^{1} \mathrm{eV} / c^{2}, a \sim 10^{-6} \mathrm{~m}$, respectively. In addition, we will calculate the number of particles in the BEC for the chosen cases. Since the elastic cross section (at low energies) is a quadratic function of the scattering length $\sigma=4 \pi a^{2}$ (Cohen et al $1977 \mathrm{~b}$ ) then our results entail an elastic cross section equal to $\sigma \sim 10^{-11} \mathrm{~m}^{2}$. Finally, it will be shown that the ensuing statistical errors in the deduction of the values for $a$ and $m$ are five and twenty four percent, respectively.

Summing up, the main result from our work states that the current observational data allow us to deduce the microscopic properties of a dark matter halo modeled as a BEC.

\section{Dark Matter and BEC}

\subsection{Gravitational Interaction and Trapping Potential}

As mentioned before we consider $N$ dark matter particles each one of them with mass $m$ and scattering length $a$ and forming a spherical body with radius $R$. This symmetry is a consequence of the assumption that all kind of external interactions upon the halo are neglected in the present work and, therefore, no privileged direction appears and hence spherical symmetry is the only possible option for the geometry of the halo.

The usual work in this context considers the GrossPitaevskii-Poisson equation, nevertheless the analytical case requires (due to the fact that there are no exact solutions to this situation) an initial assumption for the mass density of the dark matter and the choice is usually a Gaussian function (Chavanis 2011). Concerning this aforementioned condition we proceed to provide a physical argument that explains this functional structure as a consequence of the self-gravitational interaction of the halo.

Consider a spherical body of mass $M$ and radius $R$ the one has a small cavity along the diameter coincident with the $z$-axis. In addition, it has a density function with spherical symmetry, i.e., it is only a function of the radial variable. Notice that in the present situation the density has a non-compact support. We now proceed to prove that it has to be depicted by a function such that its Taylor expansion about the center of the body renders a series, not a polynomial. Indeed, we proceed by contradiction, therefore, let us assume that

$\rho(r)=\rho(0)+\sum_{n=1}^{\infty} \rho^{(n)}(0) r^{n} / n !$

Such that $\exists s \in \mathbb{N} \ni \rho^{(n)}(0)=0, \forall n \geq s$. This condition implies that around the center of the body the density is a polynomial and not a series. Therefore

$r \rightarrow \infty \Rightarrow \lim \rho(r) \rightarrow \infty$

Clearly, this is a non-physical situation and it implies that the density cannot be depicted by a polynomial. In other words, $\forall l \in \mathbb{N}, \exists s \in \mathbb{N}$ such that $\rho^{(s)}(0) \neq 0, s \geq l$, i.e., it is a series. Physically the meaning of this result is that far away from the center of the body the density vanishes, a fact impossible to achieve with a polynomial.

We now proceed to find an approximate expression for the motion of our particle moving along the diameter coincident with the $z$-axis. In order to do this let us consider one condition upon $\rho(r)$, namely, its global maximum lies at $r=0$. We may fathom this condition from a physical perspective noting that, from an intuitive point of view, gravity entails that we expect the maximum of the density to be at the geometrical center of the body.

A particle of mass $m$ moves along this cavity and we determine the classical motion equation for $m$. The coordinate system has its origin at the geometrical center of the body, and we resort to spherical coordinates. Then the motion equation reads

$m \frac{d^{2} r}{d t^{2}}=-G \frac{m M(r)}{r^{2}}$.

Where $M(r)$ denotes the mass inside the sphere of radius $r$. Due to our assumptions

$M(r)=\frac{4 \pi}{3} \rho(0) r^{3}\left[1+3 \sum_{n=2}^{\infty} \frac{\rho^{(n)}(0)}{\rho(0) n !(n+3)} r^{n}\right]$.

The equation of motion reads

$\frac{d^{2} r}{d t^{2}}+\frac{4 \pi}{3} G \rho(0) r\left[1+3 \sum_{n=2}^{\infty} \frac{\rho^{(n)}(0)}{\rho(0) n !(n+3)} r^{n}\right]=0$. 
Since the series in our last expression exists then we may define

$f(r) \equiv \sum_{n=2}^{\infty} \frac{\rho^{(n)}(0)}{\rho(0) n !(n+3)} r^{n}$

As a consequence of these arguments we have that $\forall \delta>0, \exists l \in \mathbb{N}$ such that

$\left|f(r)-\sum_{n=2}^{s} \frac{\rho^{(n)}(0)}{\rho(0) n !(n+3)} r^{n}\right|<\delta, \forall s \geq l$.

This last condition implies

$\left|\sum_{n=s+1}^{\infty} \frac{\rho^{(n)}(0)}{\rho(0) n !(n+3)} r^{n}\right|<\delta, \forall s \geq l$.

If $\delta_{1}=10^{-1}$, then $\exists l_{1} \in \mathbb{N}$ such that

$\left|\sum_{n=s+1}^{\infty} \frac{\rho^{(n)}(0)}{\rho(0) n !(n+3)} r^{n}\right|<10^{-1}, \forall s \geq l_{1}$.

This last expression entails an inequality for our equation of motion, indeed

$-\frac{d^{2} r}{d t^{2}} \leq \frac{4 \pi}{3} G \rho(0) r\left[\frac{13}{10}+3 \sum_{n=2}^{l_{1}} \frac{\rho^{(n)}(0)}{\rho(0) n !(n+3)} r^{n}\right]$

At this point we consider that our density function has the following characteristic

$\left|\frac{\rho^{(n)}(0)}{\rho(0)}\right| \sim \frac{1}{R^{n}}$

In this last expression $R$ is the characteristic radius of the region comprising most (at least 87 percent) of the particles. A word of warning is, at this point, noteworthy. Indeed, it can be easily checked that this feature is shared by several non-compact (integrable over the whole space) functions, for instance, Gaussian, Lorentzian functions and also all the even eigenfunctions of a harmonic oscillator.

Since the motion of our test particle takes place within the region defined by $r \in[0, R]$ then the ensuing equation of motion has the following approximate expression

$\frac{d^{2} r}{d t^{2}}+\frac{4 \pi}{3} G \rho(0) r\left[\frac{13}{10}+3 \sum_{n=2}^{l_{1}}\left(\frac{r}{R}\right)^{n}\right]=0$
Notice that the term with the factor 13/10 defines a three dimensional harmonic oscillator and the additional ones may be understood as perturbations to it, at least in those cases in which $r<R$. The corresponding frequency is given by

$\omega_{(0)}=\sqrt{\frac{52 \pi G \rho(0)}{30}}$.

This expression tells us that in a very rough approximation the motion of $m$ is related to an isotropic harmonic oscillator whose frequency depends upon the central density. With this classical result we now quantize our system and consider the gravitational effects of $N-1$ particles of mass $m(M=(N-1) m)$ upon our particle of, also, mass $m$. The corresponding timeindependent Schrödinger equation is then

$E \psi=-\frac{\hbar^{2}}{2 m} \nabla^{2} \psi+\frac{m \omega_{(0)}^{2}}{2} r^{2} \psi$.

Clearly, the ground state of this last equation is a Gaussian function (Cohen et al 1977a). Since the Hartree approximation is introduced then all the particles are described by a Gaussian wavefunction and therefore the mass density shares this feature. In other words, these previous arguments show us that this ubiquitous Gaussian Ansatz can be understood as an approximation in which gravity defines a threedimensional harmonic oscillator. There is an additional lesson to be elicited from these arguments, namely, the self-gravitational effects of the halo can be, in a first and rough version, be considered as defining an external trapping isotropic harmonic oscillator.

Furthermore we may obtain the radius (radius means the distance from the center of the halo such that the derivative of the rotational speed of galaxies vanishes). It is noteworthy to mention that within this region lie most of the particles, i.e., 87 percent of them. Indeed, notice that the ground state wave function related to (14) is a Gaussian function and it provides us the mass density

$$
\begin{aligned}
& \rho(r)=\frac{m N}{\sqrt{\pi^{3}} l^{3}} \exp \left(-r^{2} / l^{2}\right), \\
& l=\sqrt{\frac{\hbar}{m \omega_{(0)}}}, \\
& \rho(0)=(0.16) \frac{m N}{l^{3}} .
\end{aligned}
$$


Joining this definition and the relation for $l$ and the frequency entails

$\omega_{(0)}=\sqrt{\frac{0.9 G m N}{l^{3}}}$.

\subsection{Mathematical model}

We now take into account the case in which a nonvanishing scattering length is present in the system as an essential element. As mentioned before, the self-gravitational interaction of the dark matter halo will be considered as an isotropic harmonic oscillator and therefore our system is reduced, in its mathematical analysis, to the case of a terrestrial BEC (Pethick and Smith 2004).

According to our interpretation of the self-gravitational interaction of the halo as an external isotropic three-dimensional harmonic oscillator we have that the corresponding many body-Hamiltonian, for the situation of a dilute gas, is given by

$$
\begin{aligned}
\hat{H}= & \sum_{i=1}^{N}\left[\frac{p_{(i)}^{2}}{2 m}+\frac{m \omega_{(0)}^{2}}{2} r_{(i)}^{2}\right] \\
& +U_{(0)} \sum_{i<j} \delta\left(\vec{r}_{(i)}-\vec{r}_{(j)}\right) .
\end{aligned}
$$

In this last expression the term $U_{(0)}=4 \pi \hbar^{2} a / m$ contains the information, under the assumption of very low energies and dilute gas, of the interaction between two particles. In other words, the present model assumes, due to the fact that we have a very dilute gas, that only pairwise interactions are relevant for the dynamics of the system (Pethick and Smith 2004). Here $a$ denotes the scattering length of our particles. In this $\mathrm{so}^{-}$-called mean-field treatment all short-wavelength degrees of freedom have been integrated out (Ueda 2010).

The time-independent equation in the context of the Hartree approximation is the so-called Gross Pitaevskii equation and reads

$\mu \psi(\vec{r})=\left[-\frac{\hbar^{2}}{2 m} \nabla^{2}+\frac{m \omega_{(0)}^{2}}{2} r^{2}+U_{(0)}|\psi(\vec{r})|^{2}\right] \psi(\vec{r})$,

$N=\int|\psi(\vec{r})|^{2} d \vec{r}$

In the equation of motion $\mu$ denotes the chemical potential. Since $\rho(\vec{r})=m|\psi(\vec{r})|^{2}$ we may cast (20) in the following form $\mu \psi(\vec{r})=\left[-\frac{\hbar^{2}}{2 m} \nabla^{2}+\frac{m \omega_{(0)}^{2}}{2} r^{2}+\frac{U_{(0)}}{m} \rho(\vec{r})\right] \psi(\vec{r})$.

The lack of analytical solutions in this realm Lieb 2005) obliges us to introduce some approximation and at this point it is noteworthy to comment the assumptions that can be found in the literature and compare them to those present here, since they could, at first sight, be considered different, nevertheless they are equivalent.

The so-called Gross-Pitaevskii-Poisson system is taken as the fundamental point for the description of the situation. This means (20), or its equivalent version in the form of the Madelung formalism, plus the equation explaining the behavior of the gravitational potential $\Phi(\vec{r})$, i.e., $\nabla^{2} \Phi(\vec{r})=4 \pi G \rho(\vec{r})$. The absence of analytical solutions implies that an approximation has to be introduced, and this is done by means of the Gaussian Ansatz (Chavanis 2011). Afterwards, with this assumption the energy of the system is deduced as well as all its ensuing consequences, among them the size of the halo, etc. This is, in very few words, the logic and conditions found in the literature in this context.

We now proceed to explain the assumptions used in the present work. The gravitational effects, as explained before, are taken into account in the form of an effective three dimensional harmonic oscillator whose frequency hinges upon the mass and number of particles in the BEC, see (13), but not on the scattering length. This fact entails not only that we do not need the Gross-Pitaevskii-Poisson system, just the Gross-Pitaevskii equation, but also that the situation is, in its mathematical analysis, reduced to the case of a terrestrial BEC trapped by the usual means (Pitaevskii and Stringari 2003).

The second assumption concerns the introduction of the scattering length as a fundamental element of the description of the system. This is done taking (20) and noting that

$|\psi(\vec{r})|^{2} \psi(\vec{r})=\rho(\vec{r}) \psi(\vec{r}) / m$

Then the Gaussian structure for the density is introduced and expanded in terms of a Taylor series, keeping terms up to second order in $r$. The term depending quadratically upon $r$ is added to the term emerging from the gravitational part, i.e., the term in (22) whose coefficient is $\omega_{(0)}$. The resulting expression is then

$\tilde{\mu} \psi(\vec{r})=-\frac{\hbar^{2}}{2 m} \nabla^{2} \psi(\vec{r})+\frac{m \omega^{2}}{2} r^{2} \psi(\vec{r})$, 
$\omega^{2}=\omega_{(0)}^{2}-2 \frac{N U_{(0)}}{m \sqrt{\pi^{3}} l^{5}}$,

$\tilde{\mu}=\mu-\frac{N U_{(0)}}{\sqrt{\pi^{3}} l^{3}}$.

Here $\tilde{\mu}$ is the effective chemical potential the one suffers a modification due to the presence of the pairwise self-interaction present in the system. Expression (24) is our equation of motion for the analysis of the halo. Clearly, it is a three-dimensional harmonic oscillator in which the frequency is now modified due to the presence of a non-vanishing scattering length. If we take a closer look at the study of the size of a BEC trapped by a harmonic oscillator and in which the interaction among the particles is introduced we may find that a perturbational approach entails that the system corresponds to a harmonic oscillator in which the corresponding frequency is modified due to the presence of the scattering length (Baym and Pethick 1996). In this sense the approximation encoded in (24)-(25) is equivalent to the perturbational approach found in the literature, at least in the sense that both of them are three-dimensional harmonic oscillators.

Let us now explain some additional differences between the usual treatment (Böhmer and Harko 2007) and our model. In the literature around the mathematical analysis of a static gravitational bounded BEC the introduction of the Thomas-Fermi approximation plus the condition of a polytropic equation of state ends up with the so-called Lane-Emden equation (Böhmer and Harko 2007) the one implies a density distribution for the dark matter provided by the spherical Bessel function of 0 order., i.e., $\rho_{d m} \sim \sin (k r) /(k r)$.

The Thomas-Fermi approximation requires the fulfillment of a condition involving the total number of particles $(N)$, the scattering length $(a)$ plus the characteristic length $(l)$ related to the trapping potential, i.e., $(N a) / l>1$ (Pethick and Smith 2004). In the current works in this context the use of Thomas-Fermi condition is assumed, before the corresponding values for $N, a$, and $l$ are known. As mentioned before we do not resort to this approximation since our goal is the deduction of these parameters and, in consequence, we have no information allowing us to introduce it. In this sense our approach is a more general one. At this point a word of warning is required. Indeed, the ThomasFermi approximation neglects the kinetic energy when compared to the interaction or oscillator energies. In other words, there is no kinetic energy in this approach. In our model the situation is translated into the case of an effective harmonic oscillator and, in consequence, due to the structure of the corresponding Hamiltonian the expectation values of the potential and kinetic energies are equal (Cohen et al 1977a). This last argument tells us that if we consider the case of vanishing kinetic energy in our procedure, then we also demand implicitly (due to the fact that they are equal) vanishing potential energy and therefore the whole energy vanishes. In other words, the comparison against the case deduced resorting to Thomas-Fermi cannot be done.

\section{Microscopic and macroscopic variables}

We now proceed to analyze the relationship between two different sets of parameters related to the halo, namely, microscopic and macroscopic features. The idea is to consider the conditions related to the mechanical equilibrium of the halo, its relation to the tangential velocity of rotating galaxies, and, finally, how a beam of light is deflected by dark matter. These three aforementioned conditions have a macroscopic character and will be cast as functions of the microscopic ones, i.e., mass and scattering length of the dark matter particles plus the number of particles contained in the halo. In this manner we will have posed three expressions for the three microscopic variables in terms of characteristics which have an observational possibility.

\subsection{Macroscopic parameters}

\subsubsection{Mechanical equilibrium}

The first point concerns the expression defining the condition of mechanical equilibrium for the BEC. Indeed, gravity tends to collapse the halo and this behavior faces a pressure which is a consequence of Heisenberg's Uncertainty Principle and of the movement of the particles of the halo. Mechanical equilibrium emerges when the corresponding pressures of these two processes are equal.

The energy of the system due to $N_{(0)}$ particles in the ground state is given by

$E_{(0)}=\frac{3}{2} \hbar \omega N_{(0)}$

In this last expression the frequency corresponds to (25) and it does not neglect the kinetic energy, i.e., our formalism does not resort to the Thomas-Fermi approximation (Pethick and Smith 2004).

Since our system is equivalent to a terrestrial BEC trapped by a three-dimensional isotropic harmonic oscillator then we must remember that even in the case 
of vanishing temperature the presence of $a \neq 0$ implies, unavoidably, that excited states have to be also populated, a physical consequence of the presence of a finite coherence length (Pitaevskii and Stringari 2003).

The literature in the realm of dark matter as a BEC usually neglects the effects of the thermal cloud (Chavanis 2011; Böhmer and Harko 2007). The quest for a more realistic formalism has led us to introduce the depletion term which is the variable containing the information of the number of particles populating excited states, namely

$N_{(e)}=\frac{8}{3} N\left(\frac{N a^{3}}{\pi V}\right)^{1 / 2}$.

This last result stems from the case in which the BEC is trapped by walls, not by a harmonic oscillator. In order to fathom better the reasons for its use for cases in which a harmonic oscillator is present let us comment that there is, approximately, one excited particle per volume $\xi^{3}$, being $\xi$ the so-called coherence length (Pethick and Smith 2004). The definition of coherence length under the presence of walls reads: it is the distance over which the wave function rises from zero at the wall to close to its bulk value. Mathematically this is written as: $\frac{\hbar^{2} m}{\xi^{2}}=n U_{(0)}$. Under the presence of a trapping potential with the structure of a harmonic oscillator we notice that this condition shall include the energy of each particle emerging from the interaction with the trap, namely: $\frac{\hbar^{2} m}{\xi^{2}}=n U_{(0)}+m \omega^{2}(\xi-R)^{2} / 2$. Assuming that the energies of the self-interaction and that from the trap are similar in their order of magnitude, $n U_{(0)} \sim m \omega^{2}(\xi-R)^{2} / 2$, then $\frac{\hbar^{2} m}{\xi^{2}}=2 n U_{(0)}$, and hence in this new situation the coherence length has the same order of magnitude that in the case of walls as trapping potential, as a matter of fact the difference is only a factor of $\sqrt{2}$. Therefore the depletion terms is provided by

$N_{(e)}=\frac{2^{7 / 2}}{3} N\left(\frac{N a^{3}}{\pi V}\right)^{1 / 2}$.

This last argument proves that in a very simplified scheme the structure of the coherence length, for the case in which a non-trivial trapping potential is present, has the same structure as the homogeneous situation.

These excited particles provoke pressure which has to be included in the calculation of the mechanical equilibrium. Clearly, $N_{(e)}$ particles in the thermal cloud induce a larger pressure than the same number of particles in the ground state, the reason is related to the fact that in excited states they have a larger momentum. Therefore we may conjecture that the inclusion of the depletion term will imply, among other possibilities, halos with larger masses than those appearing in works in which all particles populate the ground state. In addition, the halo will also have a larger volume, here the argument lies upon the fact that excited states related to bound particles occupy a larger region than those in the ground state. In other words, the presence of the depletion term allows us to conjecture that here we will obtain larger and more massive halos.

In order to obtain the energy of those particles in excited states we resort to (24) which describes the BEC as a Hamiltonian with an effective three-dimensional harmonic oscillator potential. Of course, a particle in the first excited state of this system will have the energy $\epsilon_{(1)}=\frac{5}{2} \hbar \omega$ and, in consequence, our approximation is that the thermal cloud has an energy equal to

$E_{(1)}=\frac{5}{2} \hbar \omega N_{(e)}$

The total energy is provided by

$E_{(T)}=E_{(0)}+E_{(1)}$.

The pressure due to this energy is given by the expression $P_{(c)}=-\frac{\partial E_{(T)}}{\partial V}$ and since we have a spherically symmetric body of volume $V$ this parameter is a function of the characteristic length of our harmonic oscillator and we must deduce it. In order to do this we consider the content of dark matter at those points at which the rotational speed of a galaxy takes its maximum value, namely, the size of a sphere such that any star located on its surface has a rotational speed whose derivative (with respect to the distance to the geometric center of the halo) vanishes. The physical reason for this particular choice will be thoroughly explained in the next subsection in connection with one of our extant astronomical readouts.

The functional relation between speed and dark matter is given by

$v^{2}(r)=\frac{G M(r)}{r}$

Here $M(r)$ denotes the total dark matter contained in a sphere of radius $r$, namely, $M(r)=M_{(0)}(r)+$ $M_{(e)}(r)$, the sum of the mass of particles in the ground state and excited states, respectively. Since our model is, mathematically, a three-dimensional harmonic oscillator, then (assuming that only the first excited state is populated) we have that 


$$
\begin{aligned}
& M_{(0)}(r)=\frac{4 m N_{(0)}}{\sqrt{\pi} l^{3}} \int_{0}^{r} z^{2} \exp \left(-z^{2} / l^{2}\right) d z, \\
& M_{(e)}(r)=\frac{8 m N_{(e)}}{3 \sqrt{\pi} l^{5}} \int_{0}^{r} z^{4} \exp \left(-z^{2} / l^{2}\right) d z .
\end{aligned}
$$

The condition $d v^{2} / d r=0$ becomes

$$
\begin{array}{r}
M_{(0)}(r=R)+M_{(e)}(r=R)=\frac{4 m}{\sqrt{\pi} l^{3}}\left(N_{(0)} R^{3}\right. \\
\left.+\frac{2 N_{(e)}}{3 l^{2}} R^{5}\right) \exp \left(-R^{2} / l^{2}\right) .
\end{array}
$$

Integration by parts of expressions (33) and (34) entails that (35) is equivalent to

$$
\begin{array}{r}
\left(N_{(0)}(R / l)^{3}+\frac{2}{3} N_{(e)}(R / l)^{5}\right) \exp \left(-R^{2} / l^{2}\right)= \\
-\left(\frac{N}{2}(R / l)+\frac{N_{(e)}}{3}(R / l)^{3}\right) \exp \left(-R^{2} / l^{2}\right) \\
+\frac{N}{2 l} \int_{0}^{R} \exp \left(-z^{2} / l^{2}\right) d z .
\end{array}
$$

Concerning the integral in this last expression we may comment that it is related to the so-called probability integral $(\Phi(z))$ and among its possible representations we have the following one (Gradshtevn and Ryzhik 1980)

$\Phi(z)=\frac{2}{\sqrt{\pi}} \exp \left(-z^{2}\right) \sum_{s=0}^{\infty} \frac{2^{s} z^{2 s+1}}{(2 s+1) ! !}$.

In order to have an analytical expression we consider in the last series up to $s=3$. The theory of ultra-cold dilute bosonic gases tells us that $N_{(0)}>N_{(e)}$, introducing this condition in our ensuing algebraic equation implies that the equation to be solved reads

$-\left(\frac{R}{l}\right)^{3}+\frac{1}{5}\left(\frac{R}{l}\right)^{5}+\frac{2}{35}\left(\frac{R}{l}\right)^{7}=0$.

The solution is

$R=1.67 l$.

Therefore the content of dark matter within the sphere of radius $R$ is

$$
\begin{array}{r}
M(r=R)=\frac{4 m N_{(0)}}{\sqrt{\pi} l^{3}}\left(\frac{l^{3}}{2} \int_{0}^{1.67} \exp \left(-w^{2}\right) d w\right. \\
\left.-\frac{(1.67)}{2}(0.061) l^{3}\right) \\
+\frac{8 m N_{(e)}}{3 \sqrt{\pi} l^{5}}\left(\frac{3 l^{5}}{4} \int_{0}^{1.67} \exp \left(-w^{2}\right) d w\right. \\
\left.-\frac{(14.3)}{4}(0.061) l^{5}\right) .
\end{array}
$$

Resorting to tables (Abramowitz and Stegun 1970) we obtain that

$\int_{0}^{1.67} \exp \left(-w^{2}\right) d w=0.87$.

Finally, the sought mass reads

$M(r=R)=(0.87) m N\left[1-\frac{2^{3 / 2}}{3} \sqrt{\frac{N a^{3}}{R^{3}}}\right]$.

We now hark back to the deduction of the pressure of the condensate such that the volume is define as $V=$ $\frac{4 \pi}{3}(1.67 l)^{3}$. The ensuing result is

$$
\begin{array}{r}
P_{(c)}=\frac{6 \hbar^{2} N}{13.4 \pi m R^{5}}+\frac{6 U_{(0)} N^{2}}{13.4 \pi^{5 / 2} R^{6}} \\
+\frac{14 \hbar^{2} N}{10 \pi m R^{5}} \sqrt{\frac{2 N a^{3}}{\pi V}}
\end{array}
$$

It has to be underlined that the deduction of the energy of the first excited state as a consequence of a perturbation process related to our three-dimensional harmonic oscillator requires an explanation. Clearly, the effects of the presence of a non-vanishing scattering length for the ground state can be handled according to a perturbation approach as long as the interaction energy per particle in the ground state is smaller than the ground energy associated to the unperturbed threedimensional harmonic oscillator Hamiltonian i.e.,

$|<0,0| \hat{W}|0,0>|<\frac{3 \hbar \omega}{2}$.

In this last expression $\hat{W}$ denotes the operator related to the interaction potential of two particles in the ground state. Concerning the first excited state we have the same kind of condition behind this approximation

$|<1,1| \hat{W}|1,1>|<\frac{5 \hbar \omega}{2}$. 
Let us now address the issue concerning the gravitational attraction of the halo, a topic related to the equilibrium of a spherical body with density, pressure, velocity field, and gravitational potential $\rho, P$, and $\vec{v}$, $\Phi$, respectively. The corresponding equations for the internal structure are (Poisson and Will 2014)

$\rho \frac{d \vec{v}}{d t}=\rho \nabla \Phi-\nabla P$

$\frac{\partial \rho}{\partial t}+\nabla \cdot(\rho \vec{v})=0$

To close the system an additional piece of information is required, namely, the equation of state, i.e., the functional dependence among pressure, density, temperature, etc. The Newtonian gravitational potential for a spherical body of radius $R$ is

$\nabla \Phi(t, r)=\frac{G M(t, r)}{r}+4 \pi \int_{r}^{R} \rho\left(t, r^{\prime}\right) d r^{\prime}$

In this last expression $M(t, r)$ is the mass contained in a sphere (coincident with our body) of radius $r$. We now consider the surface of our sphere and calculate the change in this gravitational potential due to a change in the volume, a process that entails a pressure $\left(P_{(g)}=\right.$ $\left.-\frac{\partial \Phi}{\partial V}\right)$

$P_{(g)}=\frac{G M^{2}}{4 \pi R^{4}}$.

In the general situation the pressure is a nonconstant function of the radial distance (Poisson and Will 2014) and, in consequence, we must identify the value of $r$ related to (49). Since this pressure is deduced after evaluating the gravitational potential on the surface of the body then it represents the pressure on this surface. The mathematical condition determining mechanical equilibrium is the equality of our two pressures on the surface of the halo (remember that $M=m N$ ) i.e., expressions (43) and (49).

One consequence of the equality $P_{(g)}=P_{(c)}$ is related to the fact that we have deduced $R$ as a function of $m, a$, and $N$. In other words, if we have the size of a halo, by mean of any kind of astrophysical or astronomical observations, then we have the first of our required expressions. These last comments also provide a physical explanation to the choice done in the deduction of our parameter $R$. Indeed, we are forced, due to the extant astronomical observations, to deduce the content of matter and size (as function of $l$ ) of the region at which the derivative of $v^{2}(r)$ vanishes.

\subsubsection{Concordance with rotation velocities}

It can be said, without exaggerating, that dark matter is a descendant of the rotation curves of spiral galaxies (Binney and Tremaine 1987) and, in consequence, we must include the explanation of them, as a fundamental part of the present work. In this section we deduce the relationship between these curves and the dark matter content of our model.

Let us consider the tangential velocity of a star, which according to Newtonian physics is provided by

$\frac{v_{t}^{2}(r)}{r}=\frac{G M^{(T)}(r)}{r^{2}}$

In this last expression $M^{(T)}(r)$ denotes the total mass within a sphere of radius $r$, i.e., it includes baryonic and dark matter. Let us now consider the density of the involved baryonic mass of the halo $\left(\rho^{(b)}\right)$ and dark matter in its ground state and excited states, $\rho^{(0)}$, $\rho^{(e)}$, respectively (here $\rho^{(e)}$ is deduced from the wave function related to the first excited state of a threedimensional harmonic oscillator (Cohen et al 1977a))

$$
\begin{array}{r}
M^{(T)}(r)=M^{(b)}(r)+ \\
\int_{0}^{r} \int_{0}^{\pi} \int_{0}^{2 \pi}\left[\rho^{(0)}\left(r^{\prime}\right)+\rho^{(e)}\left(r^{\prime}\right)\right] r^{\prime 2} d r^{\prime} d \Omega \\
M^{(b)}(r)=\int_{0}^{r} \int_{0}^{\pi} \int_{0}^{2 \pi} \rho^{(b)}\left(r^{\prime}\right) r^{\prime 2} d r^{\prime} d \Omega .
\end{array}
$$

Expression (50) implies that

$$
\frac{d\left(v_{t}^{2}(r)\right)}{d r}=-\frac{v_{t}^{2}(r)}{r}+\frac{G}{r} \frac{d\left(M^{(T)}(r)\right)}{d r} .
$$

Let us now denote by $r=R$ (here $R=1.67 l$, see expression (39) ) the value of the radial coordinate at which (53) vanishes. Then we obtain the following condition

$$
\begin{array}{r}
v_{t}^{2}(R)=4 \pi \rho^{(b)}(R) R^{2}+\frac{4 \pi m N G}{(\sqrt{\pi} l)^{3}}[1 \\
\left.+\sqrt{\frac{32 a^{3} N}{3 \pi^{2} l^{3}}}\left(-1+\frac{2 R^{2}}{3 l^{2}}\right)\right] R^{2} \exp \left(-\frac{R^{2}}{l^{2}}\right) .
\end{array}
$$

A fleeting glimpse at this last expression allows us to understand that if we know the value of the radial coordinate at which the tangential velocity has its maximum value, then have a second expression (which is detectable) and hinges upon $m, a$, and $N$. In other words, 
up to now we have two parameters which can be measured and such that they are functions of the variables of the BEC. The last required expression is the deflection of a light beam due to the content of dark matter.

\subsubsection{Light deflection and BEC}

The goal here is to determine the deflection of a light beam due to the presence of dark matter. For a spherical body with total mass $M^{T}$, in the weak-field limit, the line element for points outside the body reads (Will 1993)

$$
\begin{array}{r}
d s^{2}=-\left(1-2 \frac{M^{(T)}}{r}\right) d t^{2} \\
+\left(1+2 \frac{M^{(T)}}{r}\right)\left(d x^{2}+d y^{2}+d z^{2}\right) .
\end{array}
$$

The origin of the coordinate system coincides with the geometrical center of our spherically symmetric halo. For the sake of clarity we now consider a light beam moving in the plane $z=0$ and such that it approaches the halo coming from $x \rightarrow-\infty$. The initial conditions entail that for the momentum of the beam we have $p^{(z)}=0,|p(y)|<<\left|p^{(x)}\right|$, and $p^{(0)} \approx p^{(x)}$, along the whole trajectory. The impact parameter is $y=R$, in other words, the beam, concerning its position on the $y$-axis has a distance equal to the size of the halo. Under these conditions the deflection angle reads (Misner et al 1973)

$\Delta \phi=-\frac{4 G M^{(T)}}{c^{2} R}$.

In this last expression $M^{(T)}$ denotes the total mass, i.e., it includes the baryonic contribution plus dark matter.

The contribution to the deflection angle stemming solely from dark matter $\left(\Delta \phi^{(d m)}\right)$ is easily calculated noting that the rotation velocity at $r=$ $R$ satisfies the relation $v^{2}(r=R)=G M^{(d m)} / R$ (Blanford and Naravan 1992). Since the mass content within the sphere of radius $R$ has been already calculated, see (42), then the corresponding deflection angle entails

$\frac{3.48 m N G}{c^{2} R}=2 \pi \frac{v^{2}(r=R)}{c^{2}}$.

Clearly, this is a third expression relating $m, N$, and $a$ with a parameter that can be detected astronomically, the right-hand side of our last result.

\section{Discussion of results and conclusions}

We now resort to our three main expressions, i.e., the equality between (43) and (49), plus (54) and (57), which are functions of $m, a$, and $N$. Clearly, we may deduce the aforementioned physical variables associated to the BEC from our work. Indeed, we have three unknowns and three equations.

In order to obtain these parameters we will use the values of the rotation velocities of some of the stars reported in the SPARC (Spitzer Photometry \& Accurate Rotation Curve) data set (Lelli, F. et al 2016). The corresponding values are given in Table 1.

In addition, a possible situation is related to the case in which there are more than one different kind of dark matter particles, a possibility that cannot be discarded from the very beginning. Nevertheless, if we assume that all these fourteen galaxies contain dark matter stemming from the same kind of particle, then the deduced values (for $a$ and $m$ ) shall not have a large spreading, in a statistical sense.

In order to have a deeper comprehension of the present predictions we proceed to calculate the standard deviation $(\sigma)$ for $m$ and $a$ as well as the ratio between the standard deviation and square of the average value of the corresponding parameter (see Table 2).

It is readily seen that the statistical error, corresponds to a twenty four percent for the mass of the dark matter particle, whereas for the scattering length it is almost five times smaller, i.e., five percent. In other words, the model provides consistent values for $m$ and $a$ in the sense that the predictions for fourteen different galaxies do not show a large spreading.

We underline the fact that in the present work we do not introduce by hand a value for any of the microscopic properties of the BEC, they are rather calculated.

A second test of the validity of our approach can be obtain noting that the formalism codified in our fundamental expression (19) requires the fulfillment of the condition of dilute gas, otherwise interactions involving more than two particles would become relevant in the description of the system. This condition is given by

$$
\left(\frac{V}{N}\right)^{1 / 3}>a
$$

Our calculations show that the condition is satisfied, as can be seen from our tables. There is another inequality to be fulfilled. Indeed, we know that $N \geq N_{(e)}$, resorting to according to (29) we have that $\left(3 \sqrt{\pi} / 2^{7 / 2}\right) N_{(e)}=N\left(N a^{3} / V\right)^{1 / 2}$ and this condition implies that $\left(3 \sqrt{\pi} / 2^{7 / 2}\right) \geq\left(N a^{3} / V\right)^{1 / 2}$. A fleeting 
Table 1

\begin{tabular}{lccccccc}
\hline \hline Galaxy & $v$ & $R$ & $\begin{array}{c}N m \\
10^{9}\end{array}$ & $m$ & $\begin{array}{c}N \\
10^{73}\end{array}$ & $\begin{array}{c}a \\
10^{-6} \\
{[\mathrm{~m}]}\end{array}$ & $\begin{array}{c}(V / N)^{1 / 3} \\
10^{-5} \\
{[\mathrm{~m}]}\end{array}$ \\
\hline N0055 & $8 \mathrm{~km} / \mathrm{s}]$ & {$[\mathrm{kpc}]$} & {$\left[\mathrm{M}_{\odot}\right]$} & {$\left[\mathrm{eV} / c^{2}\right]$} & & & \\
N0300 & 97.0 & 9.82 & 31.11 & 21.44 & 161.6 & 7.61 & 4.16 \\
N1090 & 176.0 & 11.28 & 146.90 & 16.77 & 975.9 & 4.80 & 2.63 \\
N3198 & 157.0 & 14.05 & 145.60 & 15.46 & 1049.1 & 5.84 & 3.19 \\
N6015 & 166.0 & 21.04 & 243.76 & 12.46 & 2179.4 & 6.85 & 3.75 \\
U8550 & 57.8 & 4.39 & 6.17 & 35.50 & 19.3 & 6.91 & 3.78 \\
U9037 & 160.0 & 19.46 & 209.45 & 13.07 & 1784.5 & 6.78 & 3.70 \\
DDO64 & 46.4 & 2.08 & 1.88 & 54.49 & 3.9 & 5.61 & 3.06 \\
U5005 & 97.1 & 17.97 & 71.23 & 15.41 & 514.8 & 9.47 & 5.18 \\
U5750 & 77.6 & 11.43 & 28.94 & 20.44 & 157.7 & 8.93 & 4.88 \\
U731 & 73.4 & 8.19 & 18.55 & 24.49 & 84.4 & 7.89 & 4.31 \\
N2366 & 53.7 & 4.16 & 5.04 & 37.15 & 15.1 & 7.10 & 3.88 \\
N3274 & 82.6 & 1.89 & 5.42 & 49.49 & 12.2 & 3.47 & 1.90 \\
N5023 & 82.9 & 6.15 & 17.77 & 27.41 & 72.2 & 6.24 & 3.41 \\
\hline
\end{tabular}

Table 2

\begin{tabular}{lcccc}
\hline \hline & Mean $\bar{x}$ & Standard Deviation $\sigma$ & $(\sigma / \bar{x})$ & $(\sigma / \bar{x})^{2}$ \\
\hline$m$ & $26.05 \mathrm{eV} / \mathrm{c}^{2}$ & 12.86 & 0.50 & 0.24 \\
$a$ & $6.74 \times 10^{-6} \mathrm{~m}$ & $1.49 \times 10^{-6}$ & 0.22 & 0.05 \\
\hline
\end{tabular}

glimpse to the results here contained proves that our values do fulfill this aforementioned condition.

As mentioned before the Thomas-Fermi approximation requires the fulfillment of the condition $(\mathrm{Na}) / R>$ 1 (Pethick and Smith 2004), the one involves not only $N$ but also two additional parameters. Stating that a large number of particles is tantamount to ThomasFermi is a mistake. In the literature of the relationship dark matter-BEC the validity of Thomas-Fermi condition is assumed, before the corresponding values for $N, a$, and $R$ are known. As mentioned before we do not resort to this approximation since our goal is the deduction of these parameters and, in consequence, we have no information allowing us to introduce it.

Summing up, we modeled dark matter as a BoseEinstein condensate and considered the effects of the thermal cloud. The effects of the self-gravitational interaction have been reformulated in terms of a threedimensional harmonic oscillator and the pairwise interaction present in the case of small energies has also been considered, without resorting to the ThomasFermi approximation. The main goal has been the deduction of the microscopic properties, namely, mass, number of particles, and scattering length, related to the particles comprised in the corresponding condensate. This task has been achieved introducing three macroscopical physical conditions related to the halo, i.e., mechanical equilibrium of the condensate, explanation of the rotation curves of stars belonging to LSB galaxies, and, finally, the deflection of light due to the presence of dark matter. These three aforementioned expressions allowed us to cast the features of the particles in terms of detectable astrophysical variables. Finally, the model has been contrasted against the observational data of fourteen galaxies and in this manner we obtained values for the involved microscopic parameters of the condensate. The deduced values show an error of twenty four percent for the mass of the dark matter particle and five percent in connection with the scattering length.

We may wrap up our discussion stating that the present proposal provides a deduction, within the realm of the theory of dilute ultra-cold gases, for the microscopic properties of a dark matter halo. Additional features of physical relevance remain to be analyzed in this context and the corresponding results will be published elsewhere.

Acknowledgements B. C. acknowledges CONACyT grant No. 574365 and S. G. the received UAM grant. 


\section{References}

Abazajian, K.: Phys. Rev. D 73, 063513 (2006)

Abramowitz, M., Stegun, I.A.: Handbook of Mathematical Functions with Formulas, Graphs, and Mathematical Tables. Dover Inc., New York (1970)

Baldeschi, M.R., Gelmini, G.B., Ruffini, R.: Physics Letters B 122, 221 (1983)

Baym, G., Pethick, C.J.: Phys. Rev. Lett. 76, 6 (1996)

Binney, J., Tremaine, S.: Galactic Dynamics. Princeton University Press, Princeton (1987)

Blanford, R.D., Narayan, R.: Annu. Rev. Astron. Astrophys. 30, 311 (1992)

Böhmer, C.G., Harko, T.: J. Cosmol. Astropart. Phys. 06, 025 (2007)

Chavanis, P.-H.: Phys. Rev. D 84, 043531 (2011)

Cohen-Tannoudji, C., Diu, B., Laloë, F.: Quantum Mechanics Vol. I. John Wiley and Sons, New York, (1977a)

Cohen-Tannoudji, C., Diu, B., Laloë, F.: Quantum Mechanics Vol. II. John Wiley and Sons, New York (1977b)

Dolgov, A.D., Smirnov, A.Y.: Physics Letters B 621, 1 (2005)

Gradshteyn, I.S., Ryzhik, I.M.: Tables of integrals, series and products. Academic Press, New York (1980)

Grifols, J.A.: Astroparticle Physics 25, 98 (2006)

Kolb, E.W., Turner, M.S.: The Early Universe. Addison Wesley, New York 1990

Lelli, F., McGaugh, S.S., Schombert, J.M.: Astron. J. 152, 157 (2016)

Lieb, E.H., Seiringer, R., Solovej, J.P., Yngvason, J.: The Mathematics of the Bose Gas and its Condensation. Birkhäuser-Verlag, Basel (2005)

Misner, C.W., Thorne, K.S., Wheeler, J.A.: Gravitation. Freeman Press, San Francisco (1973)

Nishiyama, M., Morita, M.A., Morikawa, M.: 2004, preprint, astro-ph/0403571

Pathria, R.K.: Statistical Mechanics. Butterworth-Heinemann, Oxford, (1996)

Pethick, C.J., Smith, H.: Bose-Einstein Condensation in Dilute Gases. Cambridge University Press, Cambridge (2004)

Pitaevskii. L., Stringari, S.: Bose-Einstein Condensation. Oxford Science Publications, Oxford, (2003)

Planck Collaboration 2015 Results XIII. Cosmological parameters Astron. Astrophys. 594, A13 (2016)

Poisson, E., Will, C.M.: Gravity: Newtonian, PostNewtonian, Relativistic. Cambridge University Press, Cambridge (2014)

Raman, C., Köhl, M., Onofrio, R., Durfee, D.S., Kuklewicz, C.E., Hadzibabic, Z., Ketterle, W.: Phys. Rev. Lett. 83, 2502 (1999)

Reid, B.A., Percival, W.J., Eisenstein, D.J., Verde, L., Spergel, D.N., Skibba, R.A., Bahcall, N.A., Budavari, T., Frieman, J.A., Fukugita, M., Gott, J.R., Gunn, J.E., Ivezic, Z., Knapp, G.R., Kron, R.G., Lupton, R.H., McKay, T.A., Meiksin, A., Nichol, R.C., Pope, A.C., Schlegel, D.J., Schneider, D.P., Stoughton, C., Strauss, M.A., Szalay, A.S., Tegmark, M., Vogeley, M.S., Weinberg, D.H., York, D.G., Zehavi, I.: Mon. Not. R. Astron. Soc. 404, $60(2010)$
Spekkens, K., Giovanelli, R., Haynes, M.P.: Astron. J. 129, 2119 (2005)

Steigman, G., Turner, M.S.: Nuclear Physics B 253, 375 (1985)

Ueda, M.: Fundamentals and New Frontiers of BoseEinstein Condensation. World Scientific, Singapore (2010)

Viel, M., Lesgourgues, J., Haehnelt, M.G., Matarrese, S., Riotto, A.: Phys. Rev. D 71, 063534 (2005)

Will, C.M.: Theory and Experiment in Gravitational Physics. Cambridge University Press, Cambridge (1993) 